\title{
Alpha Particle Emitter
}

National Cancer Institute

\section{Source}

National Cancer Institute. Alpha Particle Emitter. NCI Thesaurus. Code C19550.

unstable isotopes of high atomic number (elements of mass number from 82 up) which energetically release from their nuclei a particle consisting of two neutrons and two protons, with a positive charge $\left(2 \mathrm{e}^{+}\right)$and identical to the helium nucleus. 